\title{
MARCHE DU TRAVAIL ET ACTIVITE FEMININE EN ALGERIE
}

\begin{abstract}
:
This paper examines, though not in an exhaustive way, the part of women's activity in the Algerian labour market. We have noted the considerable evolution of this activity in recent years. It has been evolving increasingly because of the structural mutations under way, the generalisation of compulsory access of females to education, the decrease of fertility rate and the fact that women have been noticeably forcing their way in the labour market to improve family income.
\end{abstract}

\section{Mots clés}

-Emploi-Marché du travail-Chômage-Activité féminine-Population active-Travail informel

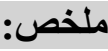

من خلال هذا المقال ، الغير مفصــل ، المتعلق بسوق

العمل و النشاط النسوي في الجزائر ، لاحظنا أن النشاط

النسوي تطور بشكل معتبر خلال السنوات الأخيـــــة .

هذا التطور سيستمر ، نظر اللتغييرات الهيكليـة الحالية ،

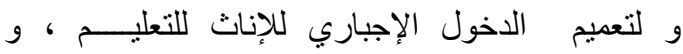

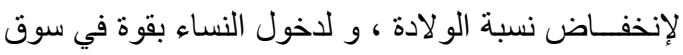

العمل لتعويض نقص مدخول العائلـــــة.

\section{BELOUCIF Ghania}

Département de Sciences Economie Université de Constantine 2

\section{Introduction :}

L'Algérie avait, jusqu'à ces dernières années, l'un des pays qui a souffert

du chômage en général et $\mathrm{du}$ chômage des femmes en particulier.

En effet, le taux de chômage a doublé entre les années 1990 et 1997 et a atteint $20 \%$ de la population active, tandis que le travail informel représente environ $25 \%$ de l'emploi du pays. 
Néanmoins, le taux de chômage a tendance à baisser pendant ces dernières années, et il avoisine les $10 \%$ ( 10,5\% exactement ) durant l'année 2016 (1). Selon l'office National des statistiques ( O.N.S ) la dernière hausse du chômage ( $10,5 \%$ en septembre 2016, contre $09,9 \%$ en avril 2016 ) a affecté essentiellement les femmes et les diplômés de l'enseignement supérieur (2). $\mathrm{L}$ e problème de la création de nouveaux emplois demeure au centre de la question du développement économique de notre pays et du marché du travail. Parmi les catégories de population exclues du marché du travail, on rencontre principalement des jeunes à la recherche de leur premier emploi et également les femmes.

Notre présent article étant justement consacré au marché du travail et à l'activité ou l'emploi féminin en Algérie, on abordera, succinctement, dans cette étude, l'évolution du marché du travail en Algérie dans une première partie, puis on consacrera la seconde partie à l'examen de l'évolution de la population active féminine.

\section{I / EVOLUTION DU MARCHE DU TRAVAIL EN ALGERIE :}

Dans cette partie, on étudie d'abord la population active et la population occupée, par sexe et par âge, pour examiner ensuite cette même population par niveau de qualification.

\section{1/ POPULATION ACTIVE ET POPULATION OCCUPEE PAR SEXE ET PAR AGE :}

On entend par population active toutes les personnes en âge de travailler et ce, qu'elles occupent déjà un emploi, ou qu'elles soient sans emploi, c'est-à-dire au chômage.

Selon l'office National des Statistiques la population active représente 12,117 millions de personne en septembre 2016 (3), alors qu'elle était de 12,092 millions de personnes en avril 2016.

(1) Quotidien 1'EST Républicain, Annaba, lundi 15/01/2017, p. 05

(2) Quotidien l'EST Républicain, op.cit., p. 05.

(3) Quotidien 1'EST Républicain, op.cit., p. 05.

Tandis que la population occupée, c'est-à-dire les personnes qui occupent déjà un emploi est estimée à 10,845 millions de personnes en septembre 2016, alors qu'elle était de 10,895 millions en avril de la même année.

TABLEAU 01: Evolution projetée de la population active par sexe (en milliers) 


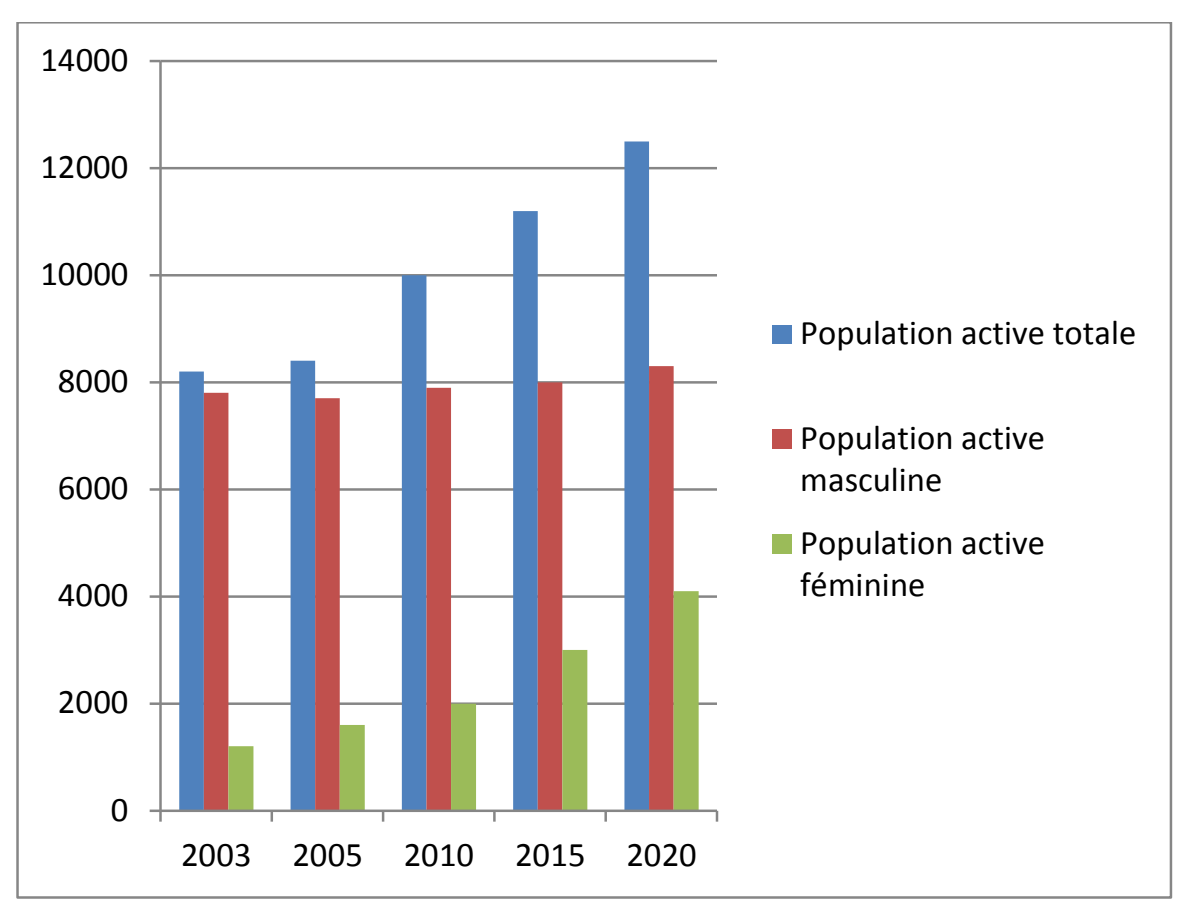

En septembre 2016, cette population occupée comprend :

- 7,558 millions de salariés, dont 4,176 millions de salariés permanents et 3,382 millions de salariés non permanents et apprentis.

- 3,133 millions d'employeurs

- 4,355 millions sont employés dans le secteur public, soit environ 40,2 \% ;

- 6,49 millions sont employés dans le secteur privé, soit 59,8\% ;

- 7,32 millions de personnes résident dans les zones urbaines ;

- 6,62 millions de personnes (soit $61 \%$ ) exercent dans les secteurs du commerce et des services ;

- 1,895 millions de personnes (soit environ 17,5 \%) dans le secteur du bâtiment et des travaux publics;

- 1,465 millions de personnes (soit 13,5\% environ) au niveau du secteur Industriel ;

- et enfin, 865.000 personnes (soit $8 \%$ ) dans le secteur agricole.

\section{1-1 : Par sexe, la population occupée se répartit ainsi :}

-Les hommes représentent 8,933 millions, soit $82,4 \%$ de cette population occupée.

- Tandis que les femmes ne représentent que 1,912 millions, soit 17,6 \% de la population occupée dans notre pays. 
Quant à la population au chômage, elle est estimée à 1,272 millions de chômeurs, dont 792.000 hommes, en septembre 2016, soit un taux de chômage de $8,1 \%$.

Alors que les femmes représentent 479.000 femmes au chômage en septembre 2016, soit un taux de chômage de 20 \%.

Il est à noter que le taux de chômage de $20 \%$ en septembre 2016 représente le taux le plus élevé de chômage chez les femmes durant les dix dernières années.

TABLEAU N² : Evolution de la population active occupée, par sexe : $1977 / 1992$

\begin{tabular}{|l|l|l|l|}
\hline & 1977 & 1982 & 1992 \\
\hline $\begin{array}{l}\text { Population occupée } \\
\text { totale } \\
\text { (hommes+femmes) }\end{array}$ & 2.336 .970 & 3.473 .900 & 4.543 .000 \\
\hline $\begin{array}{l}\text { Femmes occupées } \\
\text { dont : } \\
\text {-secteur formel } \\
\text {-Travail à domicile }\end{array}$ & 180.230 & 344.800 & 528.000 \\
\hline $\begin{array}{l}\text {-Emploi féminin } \\
\text { dans l'emploi } \\
\text { Total (\%) }\end{array}$ & 72.000 & 244.800 & 365.000 \\
\hline
\end{tabular}

Source: O.N.S., données statistiques, $\mathrm{n}^{\circ} 294$

\section{1-2 : Par âge, la population au chômage se compose ainsi :}

- Pour les personnes âgées de plus de 25 ans, le taux de chômage s'élève à $07,9 \%$, dont $05,7 \%$ sont des hommes et $16,2 \%$ des femmes.

- Pour les jeunes, âgés entre 16 et 24 ans, le taux de chômage est de 26,7 \% en septembre 2016, alors qu'il n'était que de $24,7 \%$ en avril de la même année.

\section{2/ POPULATION ACTIVE ET POPULATION OCCUPEE PAR}

\section{NIVEAU DE QUALIFICATION :}

\section{2-1: Par niveau de qualification :}

On constate que le taux de chômage, par niveau de qualification, a augmenté essentiellement chez les personnes ayant un niveau de formation supérieure. Il représente, en septembre 2016, 17,7\% chez cette catégorie de personnes, alors qu'il n'était que 13,2\% en avril 2016. 
En revanche, le taux de chômage est moins élevé chez les diplômés issus des structures de la formation professionnelle, puisqu'il ne représente, en septembre 2016, que $13 \%$ et seulement 12,1\% en avril 2016.

Quant au taux de chômage chez les personnes n'ayant aucun diplôme, il demeure faible, puisqu'il n'est que de $07,7 \%$ en septembre 2016 et $08,3 \%$ en avril de la même année.

Au total, on remarquera que :

- 45 \% des chômeurs (570.000 personnes) ne sont titulaires d'aucun diplôme.

- 28,2 \% des chômeurs (358.000 personnes) sont titulaires d'un diplôme de l'enseignement supérieur.

- 27 \% des chômeurs (343.000 personnes) sont des diplômés des structures de

la formation professionnelle.

- 66,4 \% des chômeurs sont des chômeurs de longue durée, c'est-à-dire des personnes qui sont à la recherche d'un emploi depuis plus d'une année.

- 75,3 \% des chômeurs acceptent d'occuper des emplois inférieurs à leurs niveaux de qualification professionnelle.

- 26,7 \% acceptent d'occuper des emplois pénibles ou avec un salaire inférieur à leurs qualifications $(75,8 \%)$.

\section{2-2 : Population se trouvant à la frontière du chômage :}

Cette population est composée de personnes âgées entre 16 et 59 ans, en mesure d'exercer une activité, mais n'ont pas fait de démarche pour trouver un emploi, avant le mois de septembre 2016, période à laquelle l'enquête a été réalisée par l'O.N.S. (1)

Ces personnes croient qu'elles ne pourront pas trouver du travail, ou qu'il n'existe pas d'emplois pour elles, car elles ont échoué dans leur recherche d'un emploi par le passé.

Le nombre de ces personnes recensées en septembre 2016 est de 797.000, parmi lesquelles figurent $54,6 \%$ de femmes.

Le niveau de formation de cette population est relativement faible, puisqu'on y trouve $68,8 \%$ sans aucune formation, et $61,3 \%$ ayant le niveau du cycle moyen. Parmi cette population, on trouve environ $52 \%$ qui ont moins de 30 ans, et $77 \%$ moins de 40 ans.

Après avoir étudié l'évolution du marché du travail en Algérie, d'une manière générale et examiné spécialement la population active, c'est-à-dire en âge de travailler et la population occupée réellement, par sexe et par niveau de qualification, il y a lieu d'aborder dans notre seconde partie, la question spécifique de l'évolution de la population active féminine, c'est-à-dire, la question de l'emploi féminin en Algérie. 
(1)Office National des Statistiques (O.N.S.), Quotidien l'EST Algérien, op.cit., p.05.

\section{II / L'EVOLUTION DE LA POPULATION ACTIVE FEMININE DANS LE \\ MARCHE DU TRAVAIL EN ALGERIE :}

On constate que l'activité féminine était très faible et, pratiquement, la plus

basse dans le monde, jusqu'à l'année 2000, puisqu'elle représentait environ 12 $\%$ de la population active occupée de notre pays.

Cependant, on remarque une tendance au développement de l'activité féminine durant la dernière décennie. C'est précisément cette évolution de la population active féminine en Algérie que nous allons aborder dans cette seconde partie de notre article où on étudie d'abord, les caractéristiques de la population active totale dans le marché du travail en Algérie, pour développer ensuite,

l'évolution de la population active féminine dans le marché du travail algérien.

\section{1/ LES CARACTERISTIQUES DE LA POPULATION ACTIVE TOTALE DANS LE MARCHE DU TRAVAIL EN ALGERIE :}

\section{1-1:L'évolution de la population active totale :}

La population active totale, c'est-à-dire les personnes âgées entre 15 et 59 ans, en mesure de travailler, a considérablement évolué tant au niveau de son nombre, que de sa structure par âge.

TABLEAU 3 : Evolution de la population active 1987/2000

\begin{tabular}{|l|l|l|l|l|l|l|l|l|}
\hline \multicolumn{2}{|c|}{} & \multicolumn{2}{|l|}{$\begin{array}{l}\text { Variation } \\
\text { annuel }\end{array}$} & \multicolumn{2}{|l|}{$\begin{array}{l}\text { Accroissem } \\
\text { ent annuel } \\
\text { moyen \% }\end{array}$} \\
\hline & 1987 & 1997 & 1998 & 2000 & $87 / 98$ & $\begin{array}{l}87 / 20 \\
00\end{array}$ & $\begin{array}{l}87 / \\
98\end{array}$ & $\begin{array}{l}87 / 20 \\
00\end{array}$ \\
\hline $\begin{array}{l}\text { Populat } \\
\text { ion } \\
\text { active } \\
\text { totale }\end{array}$ & $\begin{array}{l}5.341 . \\
102\end{array}$ & $\begin{array}{l}8.072 . \\
000\end{array}$ & $\begin{array}{l}8.326 . \\
624\end{array}$ & $\begin{array}{l}8.860 . \\
000\end{array}$ & $\begin{array}{l}257.0 \\
00\end{array}$ & $\begin{array}{l}263.0 \\
00\end{array}$ & $\begin{array}{l}+3, \\
93 \\
\%\end{array}$ & $\begin{array}{l}+3,15 \\
\%\end{array}$ \\
\hline $\begin{array}{l}\text { dont : } \\
\text { M. }\end{array}$ & 4.848. & & 6.761. & & 173.9 & & $\begin{array}{l}+3, \\
07\end{array}$ & \\
& 620 & & & 05 & & & 07 \\
$\%$ & & & & & & & \\
\hline F. & 492.42 & & 1.406. & & 83.05 & & $\begin{array}{l}+10 \\
\%\end{array}$ & \\
\hline Taux & 26,60 & & 27,90 & & & & & \\
\hline
\end{tabular}


MARCHE DU TRAVAIL ET ACTIVITE FEMININE EN ALGERIE

\begin{tabular}{|l|l|l|l|l|l|l|l|l|}
\hline $\begin{array}{l}\text { Brut } \\
\text { D'activ } \\
\text { ité (\%) }\end{array}$ & & & & & & & & \\
\hline $\begin{array}{l}\text { dont : } \\
\text { M. }\end{array}$ & 42,43 & & 45,70 & & & & & \\
\hline F. & 4,40 & & 9,71 & & & & & \\
\hline $\begin{array}{l}\text { Taux } \\
\text { Net } \\
\text { d'activi } \\
\text { té (\%) }\end{array}$ & 47,04 & & 48,81 & & & & & \\
\hline dont : & 85,08 & & 80,07 & & & & & \\
M. & & & & & & & & \\
\hline F. & 8,70 & & 16,96 & & & & & \\
\hline Sourc: & & & & & & & & \\
\hline
\end{tabular}

Source : O.N.S, données statistiques, $\mathrm{n}^{\circ}$ 294-305-326

On constate, par exemple, que durant la période de 1987 à 2000 cette population a augmenté de $03,93 \%$ par an, environ. Ce qui représente une offre supplémentaire annuelle de travail de l'ordre de 263.000 personnes environ, puisque cette population qui était de l'ordre de 5.341 .102 personnes en 1987, est passée à 8.860 .000 personnes en 2000 .

Cette même population a atteint 9,540 millions de personnes en 2003, soit une variation de 4.209.000 personnes, qui correspond à une demande additionnelle de 262.000 emplois par an. Cette demande additionnelle annuelle a été de l'ordre de 240.000 emplois environ.

Après avoir étudié l'évolution de la population active totale, il y a lieu d'examiner l'évolution intersectorielle de cette même population.

\section{1-2:L'évolution intersectorielle de la population active totale :}

A partir de la fin des années 1970, on a constaté une nette régression du volume de la population active et plus précisément de l'emploi, en général, au niveau du secteur industriel, qui constituait la locomotive en matière de création d'emplois.

En effet, durant ces années les emplois créés dans le secteur industriel représentaient $20 \%$ environ de l'ensemble des emplois créés.

Alors que depuis le début des années 1990, jusqu'au début de l'année 2000, le secteur industriel a connu une perte annuelle moyenne estimée à $0,6 \%$, comme le démontre le tableau ci-dessous :

TABLEAU 4: Evolution du taux annuel moyen de croissance de l'emploi, par secteur et contribution à l'emploi total, hors agriculture 
BELOUCIF Ghania

\begin{tabular}{|c|c|c|c|c|c|c|}
\hline Secteurs & & $\begin{array}{l}1967- \\
1978 \\
\end{array}$ & $\begin{array}{l}1978- \\
1986 \\
\end{array}$ & $\begin{array}{l}1986- \\
1993 \\
\end{array}$ & $\begin{array}{l}1993- \\
2000 \\
\end{array}$ & $\begin{array}{l}2000- \\
2004\end{array}$ \\
\hline \multirow{3}{*}{ Industrie } & Croissance de & 10,7 & 4,5 & 1,1 & $-1,2$ & 1,2 \\
\hline & $\begin{array}{l}\text { l'emploi par } \\
\text { périodes }\end{array}$ & 18,0 & 18,9 & 18,3 & 14,4 & 12,6 \\
\hline & $\begin{array}{l}\text { Contribution à } \\
\text { l'emploi total, hors } \\
\text { agriculture }\end{array}$ & & & & & \\
\hline \multirow[t]{3}{*}{ BTP } & Croissance de & 17,0 & 7,1 & $-1,6$ & 2,4 & 5,9 \\
\hline & $\begin{array}{l}\text { l'emploi par } \\
\text { périodes }\end{array}$ & 14,7 & 22,4 & 19,7 & 20,2 & 21,4 \\
\hline & $\begin{array}{l}\text { Contribution à } \\
\text { l'emploi total, hors } \\
\text { agriculture }\end{array}$ & & & & & \\
\hline \multirow{3}{*}{$\begin{array}{l}\text { Transport et } \\
\text { Communication }\end{array}$} & Croissance de & 7,7 & 4,3 & 5,7 & 1 & I \\
\hline & $\begin{array}{l}\text { l'emploi par } \\
\text { périodes }\end{array}$ & 6,4 & 6,1 & 6,0 & l & / \\
\hline & $\begin{array}{l}\text { Contribution à } \\
\text { l'emploi total, hors } \\
\text { agriculture }\end{array}$ & & & & & \\
\hline \multirow[t]{2}{*}{$\begin{array}{l}\text { Commerce et } \\
\text { Services }\end{array}$} & $\begin{array}{l}\text { Croissance de } \\
\text { l'emploi par } \\
\text { périodes }\end{array}$ & $\begin{array}{l}2,7 \\
28,6\end{array}$ & $\begin{array}{l}4,7 \\
21,9\end{array}$ & $\begin{array}{l}6,2 \\
20,2\end{array}$ & $\begin{array}{l}3,1 \\
27,7\end{array}$ & $\begin{array}{l}6,1 \\
29,5\end{array}$ \\
\hline & $\begin{array}{l}\text { Contribution à } \\
\text { l'emploi total, hors } \\
\text { agriculture }\end{array}$ & & & & & \\
\hline \multirow[t]{2}{*}{ Administration } & $\begin{array}{l}\text { Croissance de } \\
\text { l'emploi par } \\
\text { périodes }\end{array}$ & $\begin{array}{l}5,7 \\
32,3\end{array}$ & $\begin{array}{l}6,5 \\
30,7\end{array}$ & $\begin{array}{l}3,0 \\
35,8\end{array}$ & $\begin{array}{l}3,1 \\
37,8\end{array}$ & $\begin{array}{l}1,2 \\
36,5\end{array}$ \\
\hline & $\begin{array}{l}\text { Contribution à } \\
\text { l'emploi total, hors } \\
\text { agriculture }\end{array}$ & & & & & \\
\hline $\begin{array}{l}\text { Economie hors } \\
\text { Agriculture }\end{array}$ & $\begin{array}{l}\text { Croissance de } \\
\text { l'emploi dans } \\
\text { l'ensemble de } \\
\text { l'économie, hors } \\
\text { agriculture }\end{array}$ & 7,3 & 5,7 & 1,9 & 2,3 & 3,6 \\
\hline
\end{tabular}


On notera aussi que durant la décennie 1967 / 1977 le secteur agricole a enregistré une baisse de 170.000 emplois environ, en raison de l'exode rural considérable qui a fait chuter la contribution de ce secteur à l'emploi total de 50 \%, durant l'année 1967, à $18 \%$ en 1987.

Cependant, le programme national de développement agricole (P.N.D.A.) a permis la création de 250.000 emplois agricoles pendant les années 2000 à 2002.

Aussi, l'emploi dans l'administration algérienne a connu un développement important, en ce sens que l'administration a créé $30 \%$ du total des emplois, suite à la réalisation de nombreuses infrastructures administratives nouvelles, issues de la décentralisation administrative, mise en place à partir des années 1970 à ce jour.

Par ailleurs, le secteur rural qui occupait en 1977, environ $72 \%$ de la main d'œuvre totale, a décliné pour ne représenter en 2003 que $42 \%$ de la main d'œuvre totale et ce, en raison de l'exode rural important vers le milieu urbain. Ce qui a aggravé le chômage et les tensions au niveau du marché du travail dans notre pays.

Signalons aussi que l'emploi dans le secteur public, qui représentait $65 \%$ de la main d'œuvre en 1987 (1) a considérablement régressé, puisqu'il a enregistré une baisse de 360.000 emplois entre 1994 et 1998 (1) suite aux réformes économiques engagées par l'Etat et à la compression des effectifs dans les entreprises publiques.

En revanche, on remarquera que le secteur informel, qui représentait 58.000 emplois durant les années 1990, a vu ses effectifs doubler en l'espace de moins de dix années, puisqu'il a atteint, en 2002, 36,5\% de l'ensemble des emplois et $39 \%$ du total des emplois urbains.

Cette tendance s'accentuera, en raison de la crise économique que vit dernièrement notre pays, suite à l'effondrement des prix du pétrole, ayant entrainé le blocage des recrutements dans la plupart des secteurs de la fonction publique et ce, malgré le départ massif à la retraite d'un grand nombre de fonctionnaires, notamment, durant l'année 2016.

Tout cela, sans que le secteur privé ou les sociétés étrangères établies en Algérie n'arrivent à créer de nouveaux emplois en quantité importante.

Enfin, on constatera, malgré tout cela, un développement appréciable de l'évolution de la population active féminine dans le marché du travail de notre pays.

C'est précisément ce qui nous pousse à étudier les caractéristiques de l'évolution de la population active féminine dans le marché du travail en Algérie.

(1) CNES- 2004 
2/ LES CARACTERISTIQUES DE L'EVOLUTION DE LA POPULATION ACTIVE FEMININE DANS LE MARCHE DU TRAVAIL EN ALGERIE :

Dans cette dernière partie de notre article, on étudiera d'abord la population active féminine, pour aborder ensuite la question de l'évolution de la population active féminine.

\section{2-1 La population active féminine :}

Durant les années 1987 à 1997 la population active féminine a augmenté d'environ $10 \%$ par an, soit de 492.442 en 1987 , elle est passée à 1.406 .005 en 1998, alors que chez les hommes cette augmentation n'a été que de 3,07\%.

Cet accroissement de la population active féminine a pour origine essentielle l'accès des filles à l'école, suite à la généralisation de l'enseignement obligatoire et à l'extension du chômage, qui a touché plus de $30 \%$ de la population active totale, après la mise en place du programme d'ajustement structurel de 1994 et des mesures de compression des effectifs dans le secteur public, ( 600.000 postes de travail supprimés durant les années 1990 ), la dévaluation du dinar, ainsi que la libéralisation des prix.

Ce qui a entrainé une très forte réduction du pouvoir d'achat des salariés et poussé la population féminine à chercher du travail et obtenir ainsi une rémunération indépendante de la famille.

Aussi, l'entrée de la femme dans le marché du travail s'explique également par la diminution de la fécondité, où l'on constate que l'indice de fécondité est passé, dans notre pays, de 07,9 enfants par femme en 1970, à 07 en 1980 et à seulement 03,6 en 1996. Soit une baisse de $50 \%$ du taux de fécondité en l'espace de 26 ans.

De même que l'âge moyen au premier mariage qui était de 18 ans en 1966, est passé à 21 ans en 1977 et à 26 ans au début de l'année 2000 .

Ajouter à tout cela le niveau de qualification féminine, qui a connu un bond considérable avec l'accès grandissant de la population féminine à l'enseignement supérieur et la formation professionnelle, dans une proportion égale, ou même parfois supérieure, à celle de la population masculine.

TABLEAU $\mathrm{N}^{\circ} 05$ : Evolution du taux de scolarisation (6-15 ans) en \%, entre les années 1987 à 1998

\begin{tabular}{|l|l|l|l|}
\hline Année & 1987 & 1998 & Différence \\
\hline Ensemble & $79,9 \%$ & $81,6 \%$ & $+1,7 \%$ \\
\hline Garçons & $87,7 \%$ & $84 \%$ & $+3,7 \%$ \\
\hline Filles & $71,6 \%$ & $79,2 \%$ & $+7,8 \%$ \\
\hline
\end{tabular}

Source: ONS (RGPH : 1987 et 1998) 
On peut constater à la lecture du tableau ci-dessus, qu'en l'espace de 11 années (1987/1998), le taux de scolarisation des filles, qui était de 71,6 \% en 1987 a atteint $79,2 \%$ en 1998 , alors qu'il n'était que de $32,9 \%$ en 1962 , soit une progression significative de l'ordre de $47 \%$ en l'espace de 36 années seulement.

Il y a aussi le fait que la durée de la scolarisation des filles est plus longue que celle des garçons, avec des taux de réussite plus élevés chez les filles.

En conséquence, la population active féminine aura tendance à s'accentuer encore durant les prochaines années.

Cependant, malgré son accroissement, le taux d'activité de la population féminine demeure faible dans notre pays, comparativement à celui des pays voisins, alors que le taux de chômage y est plus élevé, comme le démontre les chiffres du tableau suivant :

TABLEAU No 06 : Activité et chômage des femmes (en \%) en Algérie, en Tunisie et au Maroc

\begin{tabular}{|l|l|l|l|l|}
\hline & $\begin{array}{l}\text { Taux d'activité } \\
\text { globale }\end{array}$ & $\begin{array}{l}\text { Taux } \\
\text { d'activité } \\
\text { féminine }\end{array}$ & $\begin{array}{l}\text { Taux de } \\
\text { chômage } \\
\text { global }\end{array}$ & $\begin{array}{l}\text { Taux de } \\
\text { chômage } \\
\text { féminin }\end{array}$ \\
\hline Algérie & $26,5 \%$ & $8,9 \%$ & $28,1 \%$ & $38,4 \%$ \\
\hline Maroc & $32,6 \%$ & $15 \%$ & $15,9 \%$ & $21,7 \%$ \\
\hline Tunisie & $29 \%$ & $12,2 \%$ & $15,3 \%$ & $20,9 \%$ \\
\hline
\end{tabular}

Source: ONS : enquête sur les niveaux de vie, 1995.

\section{2-2 L'évolution de la population active féminine :}

On examinera dans cette partie, d'une part, l'évolution de la population active féminine occupée et d'autre part, l'évolution de la population active féminine au chômage.

\section{2-2-1/ L'évolution de la population active féminine occupée :}

Comme on peut le constater à travers la lecture du tableau ci-dessous, la population féminine occupée reste faible, bien qu'elle ne cesse de progresser, puisqu'elle a atteint 11,6 \% en 1992, alors qu'en 1977 elle n'était que de 7,7 \% seulement.

TABLEAU No 07 : Evolution de la population active occupée, entre 1977 et 1992

\begin{tabular}{|l|l|l|l|}
\hline & 1977 & 1982 & 1992 \\
\hline $\begin{array}{l}\text { Population occupée totale } \\
\text { (hommes + femmes) }\end{array}$ & 2.336 .970 & 3.473 .900 & 4.543 .000 \\
\hline Femmes occupées dont : & 180.230 & 344.800 & 528.000 \\
-secteur formel & 138.000 & 244.800 & 365.000 \\
-Travail à domicile & 42.000 & 100.000 & 163.000 \\
\hline Emploi féminin dans & $7,7 \%$ & $10 \%$ & $11,6 \%$ \\
\hline
\end{tabular}


\begin{tabular}{|l|l|l|l|}
\hline l'emploi total (\%) & & & \\
\hline
\end{tabular}

ONS : Données statistiques, $\mathrm{n}^{\circ} 294$.

On signalera également que :

- La population féminine active occupée ne représentait en 2000, que 12,18 $\%$ de la population active occupée (soit 697.683 femmes), sachant que par personne "occupée » on entend celle qui est censée avoir occupé un travail rémunéré d'une heure au moins pendant la semaine du sondage.

- $84 \%$ des femmes occupaient des emplois dans le secteur formel ( secteur public ) en 1992, alors que les hommes ne représentaient que 52,3\%.

- $61,3 \%$ travaillaient au sein de l'administration, $23 \%$ dans les services, $07,3 \%$ dans l'industrie et seulement $04,5 \%$ dans le commerce en 2000.

- 163.000 femmes travaillaient à domicile en 1992, c'est-à-dire dans le secteur informel traditionnel ( artisanat, broderie, pâtisserie, etc..) ou dans un travail domestique, comme la garde des enfants ou le ménage, etc.. . Ce travail à domicile a également connu une progression assez importante, en ce sens qu'en 1977, il n'occupait que 42.000 femmes seulement.

\section{2-2-2 / L'évolution de la population active féminine au chômage :}

On observe, durant ces dernières années, un nombre sans cesse croissant de femmes au chômage ( $29,72 \%$ ), équivalant à celui des hommes $(29,78 \%)$ en 2002 .

Les deux tableaux ci-dessous font ressortir que le chômage touche essentiellement les femmes et que dans le premier tableau l'écart est de 6 points concernant le taux de chômage par sexe, avec exclusion des chômeurs qui ne cherchent pas à travailler, alors que cet écart est de 16 points, en ce qui concerne le taux de chômage par sexe, avec inclusion des chômeurs qui ne cherchent pas à travailler.

TABLEAU № 08 : Taux de chômage par sexe, à l'exclusion des chômeurs ne cherchant pas à travailler

\begin{tabular}{|l|l|l|l|}
\hline Situation & Hommes & Femmes & Total \\
\hline Occupés & 2142 & 640 & 2782 \\
\hline $\begin{array}{l}\text { Chômeurs ayant déjà } \\
\text { travaillé }\end{array}$ & 278 & 51 & 329 \\
\hline $\begin{array}{l}\text { Chômeurs n'ayant jamais } \\
\text { travaillé }\end{array}$ & 466 & 252 & 718 \\
\hline Total des chômeurs & 744 & 303 & 1047 \\
\hline Taux de chômage en $(\%)$ & $25,78 \%$ & $32,13 \%$ & $27,34 \%$ \\
\hline
\end{tabular}

Source: CENEAP : enquête « ménage », 2001.

TABLEAU $\mathrm{N}^{\circ} 09$ : Taux de chômage par sexe, avec inclusion des chômeurs ne cherchant pas à travailler 
MARCHE DU TRAVAIL ET ACTIVITE FEMININE EN ALGERIE

\begin{tabular}{|l|l|l|l|}
\hline Situation & Hommes & Femmes & Total \\
\hline Occupés & 2142 & 640 & 2782 \\
\hline $\begin{array}{l}\text { Chômeurs ayant déjà } \\
\text { travaillé }\end{array}$ & 298 & 53 & 351 \\
\hline $\begin{array}{l}\text { Chômeurs n'ayant jamais } \\
\text { travaillé }\end{array}$ & 594 & 483 & 1077 \\
\hline Total des chômeurs & 892 & 536 & 428 \\
\hline Taux de chômage en $(\%)$ & $29,4 \%$ & $45,58 \%$ & $33,92 \%$ \\
\hline
\end{tabular}

Source : CENEAP : enquête « ménage », 2001.

Enfin, on constate que depuis le début des années 1990 le chômage féminin s'oriente vers des caractéristiques nouvelles, en ce sens que, même si $62,4 \%$ des femmes au chômage sont issues du système éducatif, il y a un nombre assez élevé de femmes au foyer qui cherche désormais à trouver un emploi. Aussi, il y a environ $42 \%$ des femmes au chômage qui font partie d'une famille où il n'y a qu'une seule personne qui travaille.

En ce qui concerne l'accès des femmes aux hautes fonctions de l'Etat ou d'encadrement technique, le tableau, ci-dessous, fait ressortir en Algérie une certaine marginalisation des femmes, comparativement à 3 autres pays ( Tunisie, Maroc et Iran ) et ce, malgré le fait que les femmes algériennes ont un niveau de qualification souvent supérieur à celui des hommes, occupant les mêmes hautes fonctions.

TABLEAU $\mathrm{N}^{\circ} 10$ : Taux de féminisation des hautes fonctions dans les 4 pays

\begin{tabular}{|l|l|l|l|l|}
\hline Proportion de femmes & Algérie & Maroc & Tunisie & Iran \\
\hline Parlementaires & $3,2 \%$ & $07 \%$ & $6,7 \%$ & $4,9 \%$ \\
Cadres supérieurs et de & $5,9 \%$ & $25,6 \%$ & $12,7 \%$ & $3,5 \%$ \\
direction & $27,6 \%$ & $31,3 \%$ & $35,6 \%$ & $32,6 \%$ \\
Encadrement et & & & & \\
fonctionnement technique & & & & \\
\hline
\end{tabular}

Source: RMDH, 1998

\section{CONCLUSION :}

A travers cet article sur le marché du travail et l'activité féminine en Algérie, nous pouvons constater que, d'une manière générale, l'activité féminine est en plein essor à tous les niveaux.

Ce développement s'accentuera sans doute dans les années à venir, compte tenu des transformations structurelles que connaît notre pays dans tous les domaines et surtout en raison de la généralisation de l'accès des filles à l'enseignement et à la baisse du taux de fécondité, sans oublier que les femmes 
investissent le marché de travail en raison, soit du chômage du chef de famille, soit de l'insuffisance du revenu de la famille.

BIBLIOGRAPHIE :

(1) ONS : «Enquête nationale sur les dépenses des ménages 2000 »

(2) Boutaleb Kouider : «Le marché du travail en Algérie : le poids de l'emploi informel »

(3) Rapport des services de la présidence de la République sur « La lutte contre le chômage », compte rendu dans le quotidien « La nouvelle tribune », $1^{\text {er }}$ Oct. 2000 P.06

(4) ONS : «Données statistiques » $\mathrm{n}^{\circ} 326$ (2000)

(5) F.I.D.H : « Rapport alternatif de la FIDH au rapport initial présenté par l'Algérie au comité sur l'élimination de la discrimination à l'égard des femmes », Février 1999

(6) Données du ministère de l'éducation nationale, reprises dans le rapport du CNES sur le développement humain, 2000

(7) CNES : Rapport sur « Le regard sur l'exclusion sociale », Alger 2000

(8) BLANCHARD O.TIROLE J. 2003, «Protection de l'emploi et procédure de licenciement », rapport $n^{\circ} 44$, Conseil d'Analyse Economique, Paris, La Documentation française, p. 76.

(9) CNES, 2004, « Rapport portant évaluation des dispositifs d'emploi », Conseil National Economique et Social, Alger, p. 176 HAMMOUDA N.E., 2006, « Secteur et emploi informels en Algérie : définitions, mesures et méthodes d'estimation », MUZETTE, CHARMES, Informatisation des économies maghrébines, vol. 1, Alger, CREAD, pp. 79-117. 